\title{
Ultrasound and Color Doppler-Guided Surgery for Insertional Achilles Tendinopathy-Results of a Pilot Study
}

\author{
Håkan Alfredson ${ }^{1 *}$, Martin Isaksson ${ }^{2}$ \\ ${ }^{1}$ Department of Surgical and Perioperative Science, Sports Medicine Unit, Umeå University, Umeå, Sweden; ${ }^{2}$ Faculty of Medicine, \\ Umeå University, Umeå, Sweden. \\ Email: *hakan.alfredson@idrott.umu.se
}

Received November $20^{\text {th }}, 2013$, revised December $25^{\text {th }}$, 2013; accepted January $5^{\text {th }}, 2014$

Copyright (C) 2014 Håkan Alfredson, Martin Isaksson. This is an open access article distributed under the Creative Commons Attribution License, which permits unrestricted use, distribution, and reproduction in any medium, provided the original work is properly cited. In accordance of the Creative Commons Attribution License all Copyrights (C) 2014 are reserved for SCIRP and the owner of the intellectual property Håkan Alfredson, Martin Isaksson. All Copyright @ 2014 are guarded by law and by SCIRP as a guardian.

\section{ABSTRACT}

Background: Treatment of insertional achilles tendinopathy is known to be difficult. Eccentric calf muscle exercises and extracorporeal shockwave therapy have been proposed as the primary treatments for this condition, but surgery is indicated after failed conservative management. There is no consensus about the most efficient surgical treatment. Objectives: To evaluate a new ultrasound and color Doppler-guided surgical treatment for insertional achilles tendinopathy. Patients and methods: 24 consecutive patients (13 men and 11 women, mean age 47 years) with a long duration of insertional achilles tendon pain (median 18 months) were included in the study. The surgical procedure consisted of extirpation of the subcutaneous and retrocalcaneal bursa, scraping of the ventral distal achilles tendon and removal of prominent bone at the upper calcaneal tuberosity (Haglund's deformity). In 13 patients, the plantaris tendon was also cut and excised. VAS for pain during activity, satisfaction with the treatment result and the SF-36 score to measure quality of life were used for evaluation. Results: The mean VAS had decreased from 72 before surgery to 19 after surgery $(p<0.001)$, and 20/24 $(83 \%)$ patients were satisfied with the result and had returned to full tendon loading activity at the one-year follow-up. The SF-36 score had improved significantly. Conclusion: Ultrasound and color Doppler-guided surgical treatment seems to be a good method for treatment of chronic painful insertional achilles tendinopathy. Longer follow-up studies on larger materials are needed.

\section{KEYWORDS}

Insertional; Achilles; Tendinopathy; Bursitis; Haglund's Deformity; Ultrasound + Doppler

\section{Introduction}

Chronic Achilles tendon pain is a relatively common problem [1], often difficult to treat [2] and occur in both athletes [3] and non-active individuals [4]. The midportion of the achilles tendon is the most common location for pain $(55 \%-65 \%)$, followed by the insertion $(20 \%$ 25\%) [5].

Insertional achilles tendon problem, now commonly called insertional achilles tendinopathy, comes with posterior heel pain and a prominent tendon insertion. The symptoms are often aggravated by an increase in activity level. The diagnosis is mainly based on clinical examina-

"Corresponding author. tion, but ultrasound can be a useful tool in examining the achilles tendon insertion, bursa and bone [6].

There is more knowledge about the pathogenesis of the painful midportion achilles tendon, other than about its insertion, but some evidence indicates partially the similar pathology. In both conditions ultrasound (US) with color Doppler (CD) has shown a high blood flow in the painful tendons in contrast to tendons from pain-free individuals $[7,8]$.

Apart from the pathological distal achilles tendon itself, other tissues have been associated with insertional pain. Bursitis in the deep and subcutaneous bursae [9], a skeletal prominence located posterosuperior at the calcaneal tuberosity (Haglund's deformity) causing a tendon-cal- 
caneal impingement [10], and the presence of calcifications in the achilles tendon insertion have all been associated with posterior heel pain. The plantaris longus tendon could possibly also be associated with this condition [11].

Most patients with insertional achilles tendon pain can be managed non-operativly [4]. Rest, modified eccentric calf muscle exercises [12,13] or extracorporeal shockwave therapy [14] has been proposed as primary treatment [15]. The use of soft heel pads to raise the patient's heel-drop has also been shown to relieve pain symptoms. [15].

When conservative treatment fails, surgery is indicated. Many different surgical techniques have been described, such as extirpation of the retrocalcaneal bursa and resection of the upper calcaneus [16], and detachment of the achilles tendon at its insertion followed by removal of intratendinous calcifications [17,18]. Most methods described include tendon invasive procedures and require long postoperative rehabilitation periods. There is no consensus regarding the most efficient surgical treatment for insertional achilles tendinopathy [19].

From a parallel study, preliminary data of histochemically examined tissue samples from the subcutaneous and retrocalcaneal bursa, the upper calcaneus, fat and fibrous tissue ventral to the distal achilles tendon, collected during insertional achilles tendon surgery show rich innervation patterns especially in the subcutaneous bursae.

The aim of this 1 -year prospective study was to evaluate a new US and CD-guided non tendon invasive surgical treatment method for patients with chronic pain and advanced changes in the achilles tendon insertion.

\section{Materials and Methods}

Ethics approval was obtained from the regional ethical board for medical research at the Umeå University, Sweden. Informed consent was obtained from all patients included in this study.

\subsection{Patients}

24 consecutive patients (13 men mean age 45 years and 11 women mean age 50 years) with a long duration of insertional Achilles tendon pain (median 18 months) were included in the study (Table 1). The activity levels ranged from non actives $(n=5)$, recreational athletes $(n=$ 13) to elite level athletes $(n=6)$. All patients were Swedish, most of them from the northern part of the country. The majority of the patients had tried other treatments before surgery including rest $(n=24)$, eccentric calf muscle training $(\mathrm{n}=10)$, local sclerosing Polidocanol injections ( $(n=12)$, NSAIDs $(n=6)$, local cortisone injections $(n=3)$, ESWL $(n=1)$, all without satisfactory results.
Table 1. Characteristics of 24 consecutive patients with painful insertional Achilles tendinopathy.

\begin{tabular}{ccc}
\hline Age (years) & 47 & $(24-64)$ \\
Weight $(\mathrm{kg})$ & 78 & $(55-100)$ \\
Height $(\mathrm{cm})$ & 175 & $(158-198)$ \\
BMI $\left(\mathrm{kg} / \mathrm{m}^{2}\right)$ & 26 & $(19-39)$ \\
Duration (months) $^{* *}$ & 18 & $(5-144)$ \\
\hline
\end{tabular}

Results are expressed as mean. (Range). *** Median.

All patients had combined pathology in the Achilles insertion, with US and CD verified affection of the distal Achilles tendon, subcutaneous and retrocalcaneal bursae and a prominent calcaneal tuberosity. Thirteen patients (8 men and 5 women) also had Plantaris tendon involvement. Patients with painful intra-tendinous calcifications or bone spurs in the Achilles insertion were excluded. The surgical procedure and postoperative rehabilitation was basically the same for all patients.

\subsection{Preoperative Ultrasound and Color Doppler Examination}

Pre-operative high-resolution grey scale US and CD examination (Acuson Sequoia 512, Siemens) with a linear multi-frequency (8 - $13 \mathrm{MHz}$ ) probe was used. Examination of the Achilles insertion showed enlarged subcutaneous and retrocalcaneal bursae with high blood flow inside and outside the bursa walls. The distal Achilles tendon was thickened with structural tendon changes located ventral and central in the tendon, and there was a high blood flow inside and outside the ventral part of the tendon. A prominent upper calcaneus, and in some cases also a thickened plantaris tendon surrounded by high blood flow was found in close relation to the medial side of the Achilles tendon.

\subsection{Surgical Procedure}

The operations were done in local anesthesia $(5-10 \mathrm{ml}$ of Xylocaine $10 \mathrm{mg} / \mathrm{ml}$ with adrenaline $5 \mu \mathrm{g} / \mathrm{ml}$ ) infiltrated into the subcutaneous tissues, inside and around the superficial and deep bursae, towards the periosteum of the upper calcaneus, and on the ventral side of the distal Achilles tendon. After 10 - 15 minutes the surgical procedure was started. Through a most often lateral longitudinal skin incision about $4-6 \mathrm{~cm}$ in length, the subcutaneous tissues were visualized (Figure 1). The first step was to locate the subcutaneous bursa, between the skin and the insertion of the Achilles tendon. First the posterior part of the bursa was carefully dissected from the skin, then the anterior part was separated from the tendon. The whole bursa was removed (Figure 2). The second step was removal of the retrocalcaneal bursa. This bursa is located between the posterior smooth surface of 


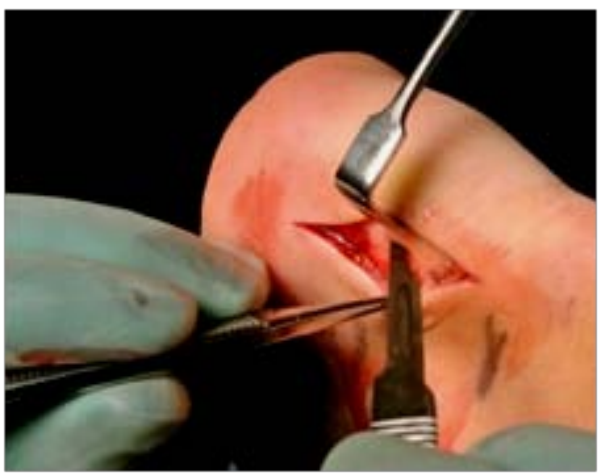

Figure 1. Straight longitudinal skin incision lateral to the Achilles insertion.

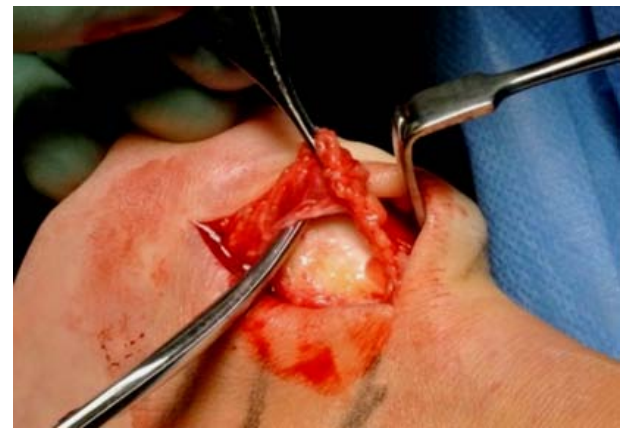

Figure 2. Careful dissection and extirpation of the superficial bursa.

the superior calcaneal tuberosity and the ventral side of the distal achilles tendon. The bursa was visible by lifting the achilles tendon posteriorly, and then the bursa was carefully dissected from the ventral tendon and removed. The third step was scraping the ventral side of the distal Achilles tendon. The surgeon carefully, with a scalpel, scraped loose the infiltrative fat tissue (including the blood vessels and accompanying nerves) outside the ventral Achilles. The fourth step was to remove the prominent upper calcaneus (Haglund's deformity). This was done by using an osteotome (Figure 3). By placing the index finger in between the tendon and upper calcaneus and dorsiflex the ankle joint, remaining impingement was excluded. The fifth step was to, via the lateral incision, go ventral to the Achilles to inspect the medial side to see if the plantaris tendon was thickened and located close to the medial Achilles. In those cases the plantaris tendon was cut, and the distal $4-5 \mathrm{~cm}$ of the plantaris was removed. Finally, the cavities (Figure 4) were flushed with 4 - $5 \mathrm{ml}$ of Xylocaine + Adrenaline, and loose bone ossicles were removed. Careful hemostasis. The skin incision was sutured with non-resorbable sutures (Figure 5).

\subsection{Postoperative Rehabilitation}

Basic steps:

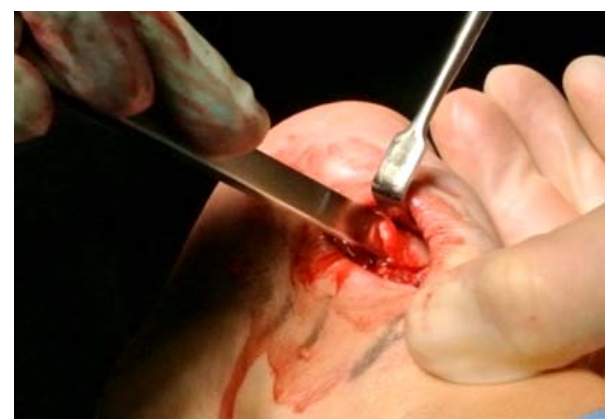

Figure 3. Removal of the Haglund deformity using an osteotome.

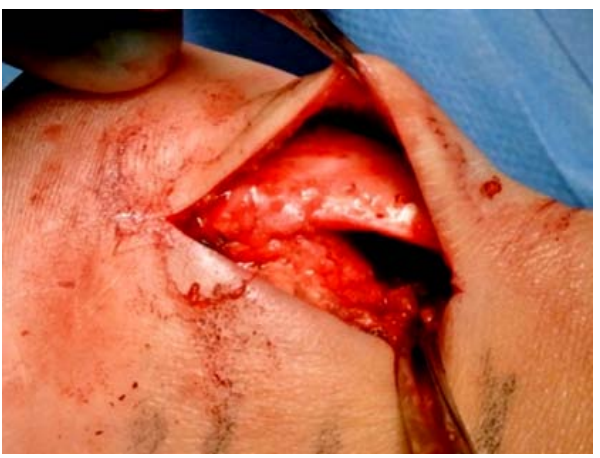

Figure 4. After bone and bursa removal there is a cavity ventral to the distal Achilles tendon.

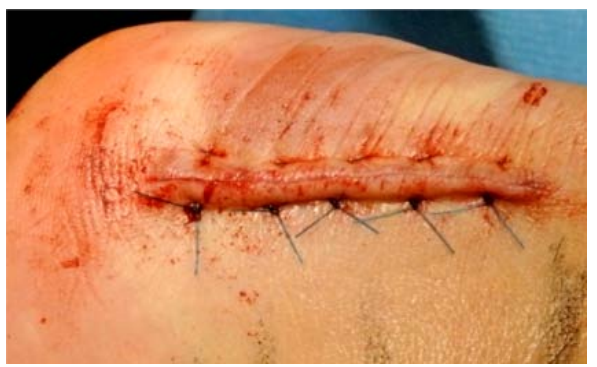

Figure 5. Skin sutures.

Day 1: Rest with elevated foot.

Weeks 1 - 6: Range of movement exercises and partial weight bearing (up to $50 \%$ of full body weight) during slow walking. Gradually increased walking distance and load on a weekly basis.

2 weeks: Suture removal.

Weeks 7 - 12: Full weight bearing walking and start of balance and coordination exercises, strength training and functional gradually increased loading.

After 12 weeks: Start slow jogging for short distances, mixed with walking (50 meters jog followed by 100 meters walk etc.) After 16 weeks: Full tendon loading sport activities.

\subsection{Evaluation}

All patients scored their level of pain during their type of 
Achilles tendon loading activity (walkers during walking, runners during running etc.) at baseline and 12 months after surgery, on a $100 \mathrm{~mm}$ long Visual Analogue Scale (VAS), where 0 is no pain and 100 is worst pain [20]. Level of satisfaction with the result of the treatment was scored one year after surgery on a scale of 0 - 100 (percentage satisfaction), where 0 is not satisfied at all and 100 is fully satisfied. Besides the one-year scores followup, there was also a telephone follow-up one year after surgery, were all patients answered the question; "Are you satisfied or not with the surgical result?”

The Swedish version of the SF-36 (The Short Form 36 Health Survey) questionnaire was used to quantify the quality of life, both from a physiological and a mental perspective, at baseline and 12 months after surgery. The SF-36 questionnaire consists of 36 questions and is divided into eight sections, each section evaluating different aspects of quality of life ( $\mathrm{PF}=$ Physical Functioning, $\mathrm{RP}=$ Role-Physical, $\mathrm{BP}=$ Bodily Pain, $\mathrm{GH}=$ General Health, VT = Vitality, $\mathrm{SF}=$ Social Functioning, RE = Role-Emotional, $\mathrm{MH}=$ Mental Health). The results are presented on a scale ranging from $0-100$, where 100 represent the highest level of quality of life in each section. The outcome of this study was compared with the outcome for the age group 45 - 54 years in the general Swedish population according to the Swedish SF-36 manual, based on previous health surveys done 19911992 in different regions in Sweden $(n=1449)[21,22]$.

\subsection{Statistical Analysis}

No data was analyzed individually, all data was analyzed on a group level. All the statistical analyses were done in the Statistical Package of Social Science (SPSS Inc., Chicago, Illinois, USA), which has been shown to be effective and accurate [23]. We used paired student's T-test to identify statistical differences in our patients $(n=24)$ before and at one year follow-up. A P-value $<0.05$ was considered to be significant.

\section{Results}

\subsection{Pain during Tendon Loading Activity}

The mean VAS (Visual analogue scale) during tendon loading activity decreased significantly $(\mathrm{P}<0.001)$ from 72 (SD 25) before surgery to 19 (SD 26) one year after surgery (Figure 6).

\subsection{Patient Satisfaction}

Mean satisfaction with treatment one year after surgery was $88 \%$ (SD $21 \%$ ). Supported by telephone follow-ups one year after surgery, 20 out of 24 patients (83\%) were satisfied with the result and had returned to full tendon loading activity.

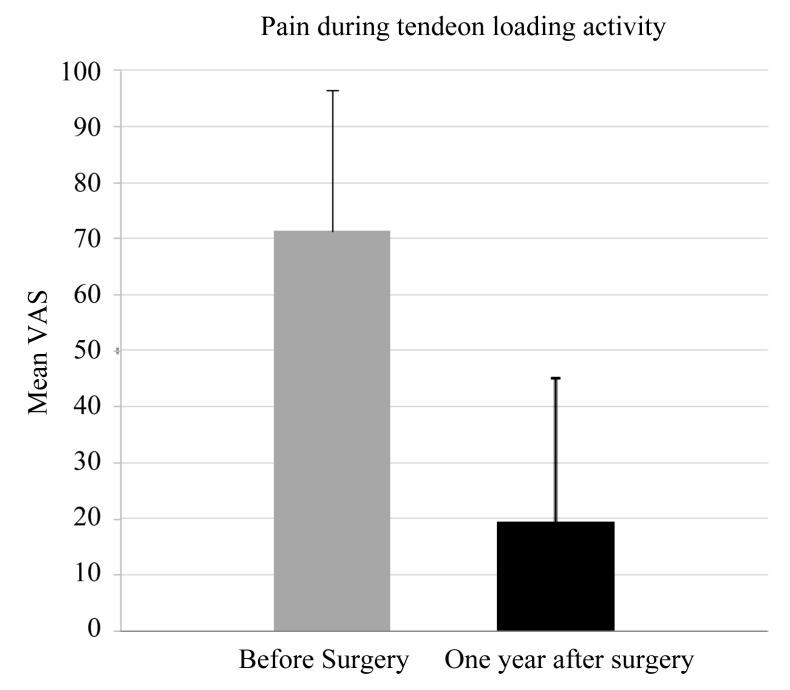

Figure 6. Visual Analogue Score (VAS) before and one year after surgery.

\subsection{Quality of Life One Year after Surgery}

The SF-36 questionnaire measuring quality of life was significantly improved at the 1-year follow-up (Figure 7). The parameters focusing on quality of life from a physical perspective were increased significantly from 42 (RP; SD 37) - 64 (PF; SD 22) before surgery to 85 (RP; SD 28) - 87 (RF; 14) at the one year follow-up (PF and RF P < $0.001)$.

The parameters focusing on quality of life from a mental perspective increased significantly from 62 (VT; SD 21) - 82 (SF; SD 21) before surgery to 71 (VT; SD 23) 93 (SF; SD 18) at the one year follow-up (VT P $=0.014$, SF $\mathrm{P}=0.018$ ).

\subsection{Complications}

One patient reported worse problems one year after surgery than before. This patient got a partial Achilles tendon rupture in the Achilles insertion 6 weeks after surgery. Worth mentioning was that this patient had previously, by a general practitioner, been treated two times with local cortisone injections in the Achilles insertion. One patient had a minor wound rupture and one patient had a streptococcus verified wound infection that was treated successfully with antibiotics.

\section{Discussion}

This one-year follow-up study on patients with chronic painful insertional Achilles tendinopathy, treated with ultrasound and Doppler-guided surgery to the superficial and deep bursa, upper calcaneus, Achilles tendon and sometimes also the plantaris tendon, showed good clinical results with a high proportion of satisfied patients back in Achilles tendon loading activity. 


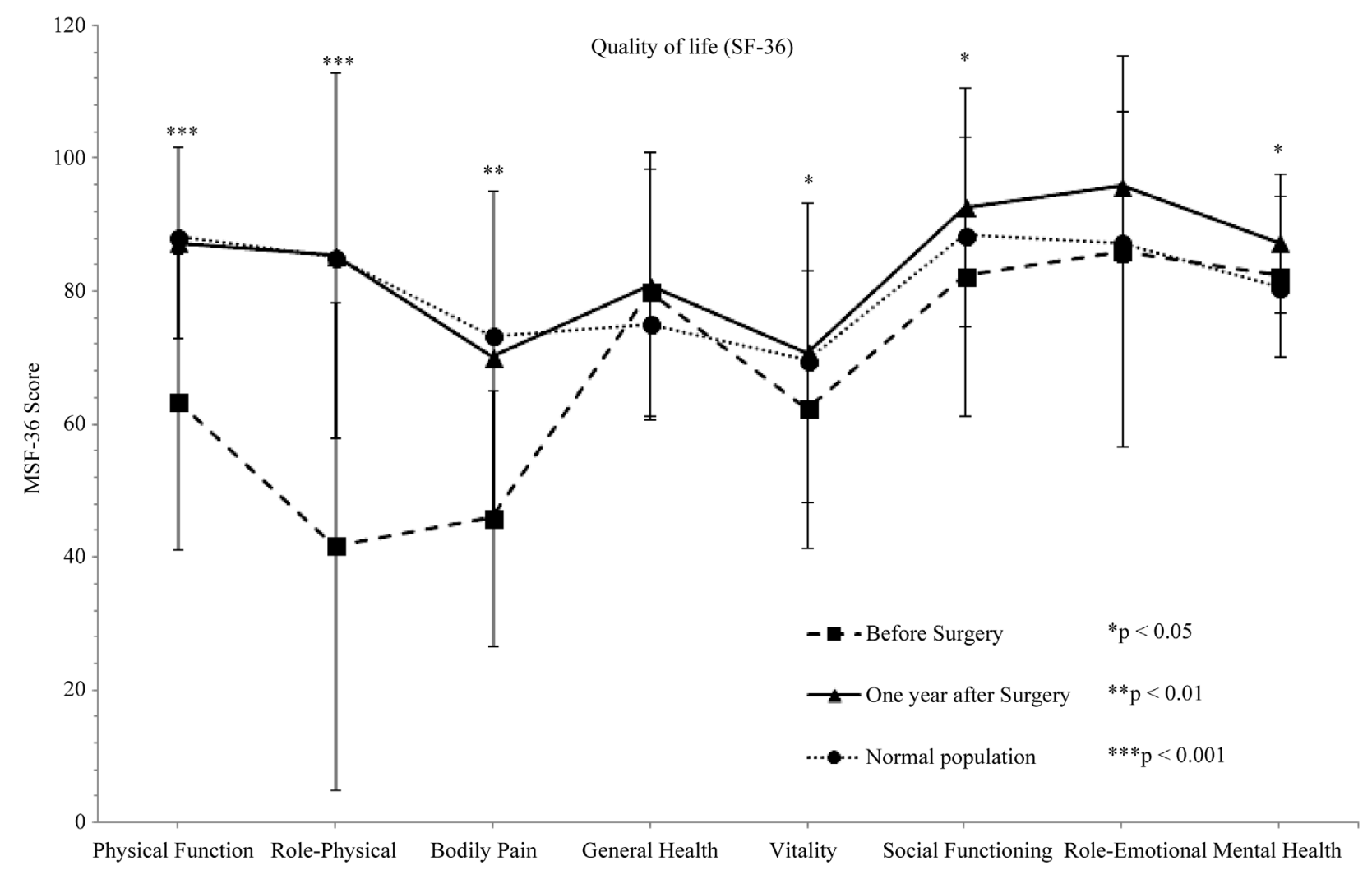

Figure 7. The SF-36 scores before and 1 year after surgical treatment, and the SF-36 score in the normal Swedish population (45 - 56 years).

It is well known that chronic painful insertional Achilles tendinopathy is difficult to treat, and a range of surgical methods have been presented for this condition. Why is this condition so difficult to treat? Why believe this is because the pathogenesis and source of pain basically is unknown. At the Achilles insertion there are three different types of tissues; bursa, bone and tendon. These tissues could alone, or together, be responsible for the pain. Preliminary results from a parallell study of ours, evaluating tissue biopsies from bursae, bone and peritendinous structures in patients with insertional pain, show a rich innervation pattern in close relation to blood vessels in especially the subcutaneous bursa. These findings are in line with previous results of biopsies taken from regions with high blood flow in chronic painful midportion Achilles and patellar tendons, where the nerves were located in close relation to blood vessels outside the painful tendons [24]. Therefore, we believe it is likely that the nerves in the bursae, and in the soft tissues outside the distal Achilles and Plantaris tendon in our patients with insertional Achilles tendon pain also is located in close relation to the regions with high blood flow found during the pre-operative $\mathrm{CD}$ examination. In our study, the findings at the pre-operative US and CD examination guided the surgical procedure. Enlarged subcutaneous and retrocalcaneal bursa with high blood flow, especially in the bursa walls, were extirpated and a prominent upper calcaneus (Haglund's deformity) was excised. In a study from 2011, we demonstrated good clinical results using a scraping procedure, targeting the regions with high blood flow and nerves outside the ventral part of the tendon in patients with painful midportion Achilles tendinosis [25]. In our current study, there was also a localized high blood flow outside the ventral part of the distal Achilles tendon pre-operatively. Therefore, the thickened distal Achilles tendon with irregular structure and high blood flow inside and outside the ventral tendon was scraped. Recent studies have demonstrated that the plantaris tendon can be subjected to tendinosis/ tendinopathy and involved in the pain mechanisms related to midportion achilles tendinosis $[11,26]$. In our study, 13 patients showed a distally thickened Plantaris tendon surrounded by high blood flow on the US and CD examination, and these findings lead to removal of the distal part of the plantaris tendon. Interestingly, in no other surgical treatment method presented in a scientific study the superficial bursa is removed. Most other studies focus on removing the retrocalcaneal bursa and upper calcaneus. We will have to wait for the final results of the immune-histochemical analyses of the different tissue types, but it might be that the major source of pain is in the superficial bursa. 
At our clinic, US and CD examination has become the most used diagnostic tool in the pre-operative evaluation of the Achilles tendon and its insertion. Ultrasound is known to be good in visualizing bone abnormalities and bursa pathology and has been shown to be one of the most accurate imaging modalities for the Achilles tendon [6]. In addition, combining US with CD provides the unique ability to study blood flow in the Achilles tendon, which has been demonstrated to be of importance when evaluating Achilles tendinosis [2,8]. However, operating an US probe is user dependent, and interpreting the images requires considerable experience, which could be a general limitation. We consider US and CD to be an effective modality to be used in the evaluation of insertional Achilles tendinopathy.

All patients in the current study were operated in local anesthesia. We have found that $5-10 \mathrm{ml}$ of Xylocaine $(10 \mathrm{mg} / \mathrm{ml})$ with adrenaline $(5 \mu \mathrm{g} / \mathrm{ml})$ injected inside and outside the bursae, around the upper calcaneus and ventral to the distal Achilles is enough for a totally pain-free operation. There are many advantages compared to general anesthesia [27] and spinal or epidural anesthesia that commonly are used for this procedure, especially since these patients are operated in prone position.

In our surgical procedure a longitudinal lateral skin incision in the Achilles tendon insertion was used, which is one of several approaches previously described [16-19, 28-30]. The lateral incision provided good access to the distal part of the tendon, the subcutaneous- and retrocalcaneal bursa, and the upper calcaneus. A disadvantage with this approach might be when examining the medially located Plantaris tendon. By saving this to the last step of the procedure, after removing the upper calcaneus, improved access to the Plantaris tendon is achieved.

We used a non-tendon invasive approach, where the upper calcaneus and retrocalcaneal bursa were removed without a tenotomy. We find the lateral approach practical, and by lifting up the tendon, bone and bursa can easily be removed. Avoiding a tenotomy enables early weight bearing tendon loading activity postoperatively. Other surgical methods for treatment of insertional Achilles tendinopathy often include longitudinal tenotomy [28] or a partial or total detachment of the Achilles tendon from the calcaneal bone [18]. However, these methods are mainly used to remove intra-tendinous calcifications or insertional bone spurs. In our study, patients with painful bone spurs and calcifications were excluded.

We used VAS during tendon loading activity, satisfaction with the treatment result and the SF-36 quality of life score for evaluation. To the best of our knowledge, quality of life as a health outcome measure in patients with insertional Achilles tendon disorders, has never been studied and evaluated before. This study demon- strated how patients with this condition experience their Quality of life before surgery, and how this type of surgical treatment significantly improved their Quality of life, both from a mental and physical perspective. Interestingly, patients with chronic painful insertional Achilles tendinopathy suffer not only physically but also mentally. This is an aspect that might be overshadowed by the physical limitations, but should be remembered and taken into account, and potentially strengthens the indication for surgical treatment.

Weaknesses in the current study are that we only had a one-year follow-up, and that this was a score and telephone follow-up, with no clinical and sonographic follow-up. The reason to this is that due to logistical problems, with patients living far away from the clinic, it was not possible to bring them in for a follow-up. However, we have planned to also have a 2- and 5-year questionnaire follow-up.

\section{Conclusion}

In conclusion, the present study showed that a high proportion of patients with chronic painful insertional Achilles tendinopathy, who undergo US and CD guided surgery with extirpation of the subcutaneous and retrocalcaneal bursa, resection of the prominent upper calcaneus, scraping of the distal tendon and sometimes also extirpation of the plantaris tendon, were satisfied with the results, experienced significantly less pain during tendon loading activity and had improved quality of life, one year after surgery.

\section{Acknowledgements}

We would like to express our sincere gratitude to Dr. Peter Larsson for help with the statistical analyses.

\section{REFERENCES}

[1] U. Waldecker, G. Hofmann and S. Drewitz, "Epidemiologic Investigation of 1394 Feet: Coincidence of Hindfoot Malalignment and Achilles Tendon Disorders," Journal of Foot and Ankle Surgery, Vol. 18, No. 2, 2012, pp. 119-123. http://dx.doi.org/10.1016/j.fas.2011.04.007

[2] H. Alfredson, "The Chronic Painful Achilles and Patellar Tendon: Research on Basic Biology and Treatment," Scandinavian Journal of Medicine \& Science in Sports, Vol. 15, No. 4, 2005, pp. 252-259.

http://dx.doi.org/10.1111/j.1600-0838.2005.00466.x

[3] K. Knobloch, U. Yoon and P. M. Vogt, “Acute and Overuse Injuries Correlated to Hours of Training in Master Running Athletes," Foot \& Ankle International, Vol. 29, No. 7, 2008, pp. 671-676. http://dx.doi.org/10.3113/FAI.2008.0671

[4] M. S. Myerson and W. McGarvey, "Disorders of the Insertion of the Achilles Tendon and Achilles Tendinitis," Journal of Bone and Joint Surgery-American Volume, 
Vol. 80A, No. 12, 1998, pp. 1814-1824.

[5] T. A. H. Jarvinen, P. Kannus, M. Paavola, T. L. N. Jarvinen, L. Jozsa and M. Jarvinen, “Achilles Tendon Injuries,” Current Opinion in Rheumatology, Vol. 13, No. 2, 2001, pp. 150-155. http://dx.doi.org/10.1097/00002281-200103000-00009

[6] K. Mahlfeld, R. Kayser, A. Mahlfeld, H. Grasshoff and J. Franke, "Value of Ultrasonography as a Diagnostic Tool of the Achilles Tendon," Ultraschall in Der Medizin, Vol. 22, No. 2, 2001, pp. 87-90.

http://dx.doi.org/10.1055/s-2001-12854

[7] K. Knobloch, R. Kraemer, A. Lichtenberg, et al., “Achilles tendon and Paratendon Microcirculation in Midportion and Insertional Tendinopathy in Athletes," American Journal of Sports Medicine, Vol. 34, No. 1, 2006, pp. 92-97. http://dx.doi.org/10.1177/0363546505278705

[8] L. Ohberg, R. Lorentzon and H. Alfredson, “Neovascularisation in Achilles Tendons with Painful Tendinosis but Not in Normal Tendons: An Ultrasonographic Investigation," Knee Surgery Sports Traumatology Arthroscopy, Vol. 9, No. 4, 2001, pp. 233-238. http://dx.doi.org/10.1007/s001670000189

[9] C. N. van Dijk, M. N. van Sterkenburg, J. I. Wiegerinck, J. Karlsson and N. Maffulli, "Terminology for Achilles Tendon Related Disorders,” Knee Surgery Sports Traumatology Arthroscopy, Vol. 19, No. 5, 2011, pp. 835-841. http://dx.doi.org/10.1007/s00167-010-1374-z

[10] S. Kang, D. B. Thordarson and T. P. Charlton, "Insertional Achilles Tendinitis and Haglund's Deformity," Foot \& Ankle International, Vol. 33, No. 6, 2012, pp. 487-491. http://dx.doi.org/10.3113/FAI.2012.0487

[11] F. Lintz, A. Higgs, M. Millett, et al., "The Role of Plantaris Longus in Achilles Tendinopathy: A Biomechanical Study,” Journal of Foot and Ankle Surgery, Vol. 17, No. 4, 2011, pp. 252-255. http://dx.doi.org/10.1016/j.fas.2010.08.004

[12] P. Jonsson, H. Alfredson, K. Sunding, M. Fahlstrom and J. Cook, "New Regimen for Eccentric Calf-Muscle Training in Patients with Chronic Insertional Achilles Tendinopathy: Results of a Pilot Study," British Journal of Sports Medicine, Vol. 42, No. 9, 2008, pp. 746-749. http://dx.doi.org/10.1136/bjsm.2007.039545

[13] E. M. Roos, M. Engstrom, A. Lagerquist and B. Soderberg, "Clinical Improvement after 6 Weeks of Eccentric Exercise in Patients with Mid-Portion Achilles Tendinopathy-A Randomized Trial with 1-Year Follow-Up," Scandinavian Journal of Medicine \& Science in Sports, Vol. 14, No. 5, 2004, pp. 286-295.

http://dx.doi.org/10.1111/j.1600-0838.2004.378.x

[14] J. P. Furia, "High-Energy Extracorporeal Shock Wave Therapy as a Treatment for Insertional Achilles Tendinopathy," American Journal of Sports Medicine, Vol. 34, No. 5, 2006, pp. 733-740. http://dx.doi.org/10.1177/0363546505281810

[15] R. Kearney and M. L. Costa, "Insertional Achilles Tendinopathy Management: A Systematic Review,” Foot \& Ankle International, Vol. 31, No. 8, 2010, pp. 689-694. http://dx.doi.org/10.3113/FAI.2010.0689

[16] M. L. Yodlowski, A. D. Scheller and L. Minos, "Surgical
Treatment of Achilles Tendinitis by Decompression of the Retrocalcaneal Bursa and the Superior Calcaneal Tuberosity,” American Journal of Sports Medicine, Vol. 30, No. 3, 2002, pp. 318-321.

[17] S. W. Keck and P. J. Kelly, "Bursitis of the Posterior Part of the Heel; Evaluation of Surgical Treatment of $18 \mathrm{~Pa}$ tients," The Journal of Bone and Joint Surgery. American Volume, Vol. 47, No. 2, 1965, pp. 267-273.

[18] N. Maffulli, V. Testa, G. Capasso and A. Sullo, "Calcific Insertional Achilles Tendinopathy-Reattachment with Bone Anchors," American Journal of Sports Medicine, Vol. 32, No. 1, 2004, pp. 174-182. http://dx.doi.org/10.1177/0363546503258923

[19] J. I. Wiegerinck, G. M. Kerkhoffs, M. N. van Sterkenburg, I. N. Sierevelt and C. N. van Dijk, "Treatment for Insertional Achilles Tendinopathy: A Systematic Review," Knee Surgery, Sports Traumatology, Arthroscopy, 2012.

[20] D. D. Price, P. A. McGrath, A. Rafii and B. Buckingham, "The Evaluation of Visual Analogue Scales as Ratio Scale Measures for Chronic and Experimental Pain,” Pain, Vol. 17, No. 1, 1983, pp. 45-56. http://dx.doi.org/10.1016/0304-3959(83)90126-4

[21] M. Sullivan, J. Karlsson and J. E. Ware, "The Swedish SF-36 Health Survey 1. Evaluations of Data Quality, Scaling Assumptions, Reliability, and Construct Validity: A Cross General Populations in Sweden,” Social Science \& Medicine, Vol. 41, No. 10, 1995, pp. 1349-1358. http://dx.doi.org/10.1016/0277-9536(95)00125-Q

[22] J. E. Ware and C. D. Sherbourne, "The MOS 36-Item ShortForm Health Survey (SF-36) 1. Conceptual Framework and Item Selection,” Medical Care, Vol. 30, No. 6, 1992, pp. 473-483. http://dx.doi.org/10.1097/00005650-199206000-00002

[23] R. X. Liu, J. Kuang, Q. Gong and X. L. Hou, "Principal Component Regression Analysis with SPSS," Computer Methods and Programs in Biomedicine, Vol. 71, No. 2, 2003, pp. 141-147. http://dx.doi.org/10.1016/S0169-2607(02)00058-5

[24] G. Andersson, P. Danielson, H. Alfredson and S. Forsgren, "Nerve-Related Characteristics of Ventral Paratendinous Tissue in Chronic Achilles Tendinosis," Knee Surgery, Sports Traumatology, Arthroscopy, Vol. 15, No. 10, 2007, pp. 1272-1279. http://dx.doi.org/10.1007/s00167-007-0364-2

[25] H. Alfredson, "Ultrasound and Doppler-Guided MiniSurgery to Treat Midportion Achilles Tendinosis: Results of a Large Material and a Randomised Study Comparing Two Scraping Techniques," British Journal of Sports Medicine, Vol. 45, No. 5, 2011, pp. 407-410. http://dx.doi.org/10.1136/bjsm.2010.081216

[26] H. Alfredson, "Midportion Achilles Tendinosis and the Plantaris Tendon," British Journal of Sports Medicine, Vol. 45, No. 13, 2011, pp. 1023-1025. http://dx.doi.org/10.1136/bjsports-2011-090217

[27] S. C. Urwin, M. J. Parker and R. Griffiths, "General Versus Regional Anaesthesia for Hip Fracture Surgery: A Meta-Analysis of Randomized Trials," British Journal of Anaesthesia, Vol. 84, No. 4, 2000, pp. 450-455. http://dx.doi.org/10.1093/oxfordjournals.bja.a013468 
[28] W. C. McGarvey, R. C. Palumbo, D. E. Baxter and B. D. Leibman, "Insertional Achilles Tendinosis: Surgical Treatment through a Central Tendon Splitting Approach,” Foot \& Ankle International, Vol. 23, No. 1, 2002, pp. 19-25.

[29] I. Elias, S. M. Raikin, M. P. Besser and L. N. Nazarian, "Outcomes of Chronic Insertional Achilles Tendinosis Using FHL Autograft through Single Incision,” Foot \&
Ankle International, Vol. 30, No. 3, 2009, pp. 197-204. http://dx.doi.org/10.3113/FAI.2009.0197

[30] M. J. DeOrio and M. E. Easley, "Surgical Strategies: Insertional Achilles Tendinopathy," Foot \& Ankle International, Vol. 29, No. 5, 2008, pp. 542-550. http://dx.doi.org/10.3113/FAI.2008.0542 